\title{
A fiber injection unit for the Keck Planet Imager and Characterizer
}

D. Mawet, J. R. Delorme, N. Jovanovic, J. K. Wallace, R. D. Bartos, et al.

D. Mawet, J. R. Delorme, N. Jovanovic, J. K. Wallace, R. D. Bartos, P. L. Wizinowich, M. Fitzgerald, S. Lilley, G. Ruane, J. Wang, N. Klimovich, Y. Xin, "A fiber injection unit for the Keck Planet Imager and Characterizer," Proc. SPIE 10400, Techniques and Instrumentation for Detection of Exoplanets VIII, 1040029 (1 September 2017); doi: 10.1117/12.2274891

Event: SPIE Optical Engineering + Applications, 2017, San Diego, California, United States 


\title{
A fiber injection unit for the Keck Planet Imager and Characterizer
}

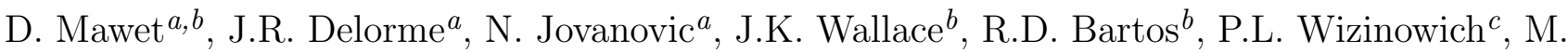

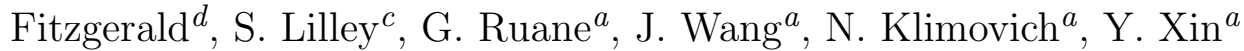 \\ ${ }^{a}$ Astronomy Department, California Institute of Technology, 1200 E. California Blvd., \\ Pasadena, CA, USA 91125; \\ ${ }^{b}$ Jet Propulsion Laboratory, California Institute of Technology, 4800 Oak Grove Drive, \\ Pasadena, CA, USA 91109; \\ ${ }^{c}$ W. M. Keck Observatory, 65-1120 Mamalahoa Hwy., Kamuela, HI, USA 96743; \\ ${ }^{d}$ Department of Physics and Astronomy, University of California-Los Angeles, 430 Portola \\ Plaza, Los Angeles, CA 90095, USA.
}

\begin{abstract}
Coupling a high-contrast imaging instrument to a high-resolution spectrograph has the potential to enable the most detailed characterization of exoplanet atmospheres, including spin measurements and Doppler mapping. The high-contrast imaging system serves as a spatial filter to separate the light from the star and the planet while the high-resolution spectrograph acts as a spectral filter, which differentiates between features in the stellar and planetary spectra. The Keck Planet Imager and Characterizer (KPIC) located downstream from the current W. M. Keck II adaptive optics (AO) system will contain a fiber injection unit (FIU) combining a high-contrast imaging system and a fiber feed to Keck's high resolution infrared spectrograph NIRSPEC. Resolved thermal emission from known young giant exoplanets will be injected into a single-mode fiber linked to NIRSPEC, thereby allowing the spectral characterization of their atmospheres. Moreover, the resolution of NIRSPEC $(\mathrm{R}=37,500)$ is high enough to enable spin measurements and Doppler imaging of atmospheric weather phenomenon. The module will be integrated and tested at Caltech before being transferred to Keck in 2018.
\end{abstract}

Keywords: Adaptive Optics, High contrast imaging, High resolution spectroscopy, Exoplanet characterization, Doppler imaging

\section{KECK PLANET IMAGER AND CHARACTERIZER}

The Keck Planet Imager and Characterizer (KPIC) consists of an upgrade to the Keck II AO system and instrument suite. KPIC features an avalanche photodiode-based infrared Pyramid wavefront sensor (IR WFS), a Boston Micromachines (BMC) high-order deformable mirror (HODM), a coronagraph and a fiber-injection unit (FIU). The key goal of KPIC is to suppress the starlight and feed the faint exoplanet light into a single mode fiber in order to spectrally characterize the planetary companion using the high resolution spectrograph (HRS), NIRSPEC. Fig.1 shows a block diagram of the KPIC instrument.

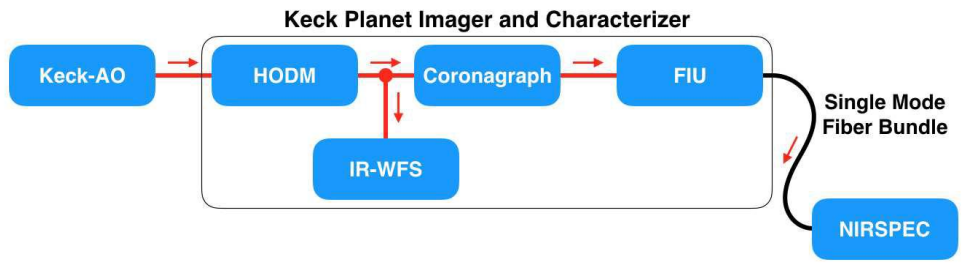

Figure 1. Block diagram of KPIC. HODM - High order DM, FIU - fiber injection unit, IR-WFS - IR wavefront sensor.

D. Mawet - E-mail: dmawet@astro.caltech.edu

Techniques and Instrumentation for Detection of Exoplanets VIII, edited by Stuart Shaklan, Proc. of SPIE Vol. 10400, 1040029 · C 2017 SPIE · CCC code: 0277-786X/17/\$18 · doi: 10.1117/12.2274891 
During spring 2017, the infrared wavefront sensor and the fiber injection unit of KPIC successfully passed a preliminary design review (PDR). Long-lead items such as the off-axis parabolas, tip-tilt mirror, and tracking camera were ordered immediately following the PDR. The FIU module will initially be aligned at Caltech before being delivered to Keck II in early 2018. We plan to implement the FIU in two phases, requiring two different units. The first FIU plate is a fast-track prototype which will not include the HODM and the coronagraph. The intended use for the FIU prototype is to send a test particle to Keck in order to learn about and optimize sub-system and operational interfaces.

A second iteration of the FIU including the high-performance hardware (DM and coronagraph) is currently being designed and is expected to follow the first plate by six to twelve months. The most recent 3D model of the second iteration of our FIU design is shown in Fig.2.
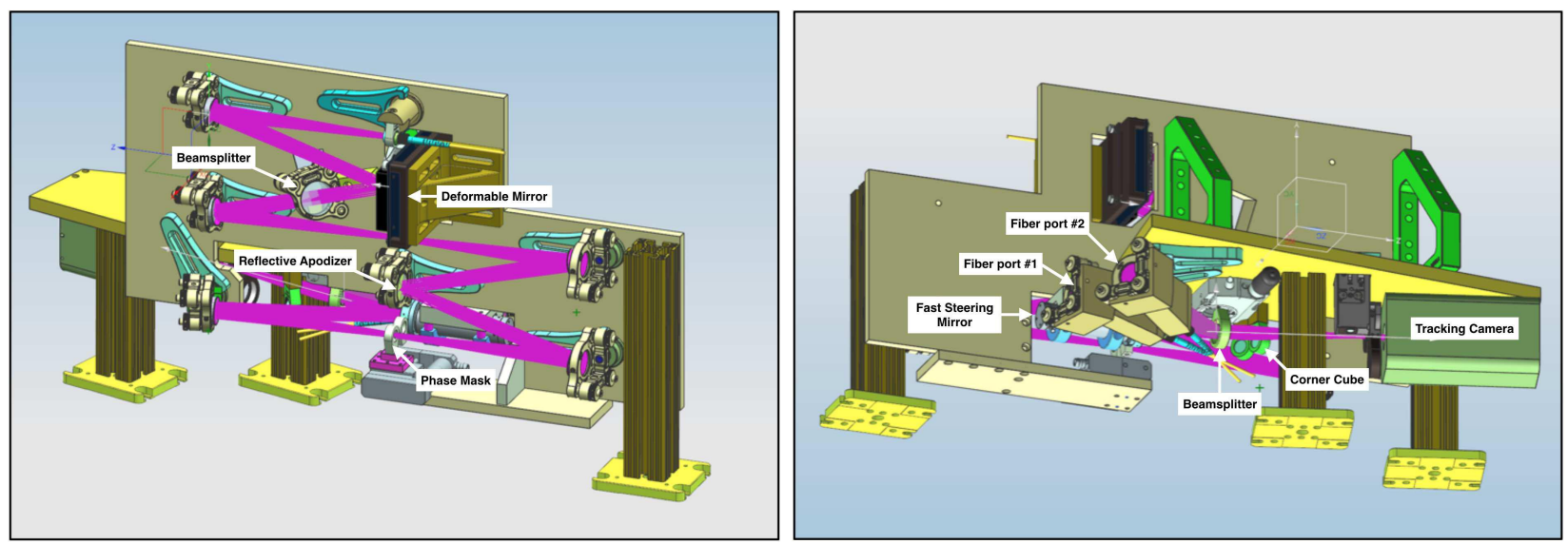

Figure 2. Front side (left) and back side (right) of a 3D model of the Keck Planet Imager and Characterizer, including the fiber injection unit. The infrared pyramid wavefront sensor is not shown.

\section{HIGH DISPERSION CORONAGRAPHY}

The KPIC FIU modules are designed to implement high dispersion coronagraphy (HDC) as outlined in Ref. 1, Ref. 2 and Ref. 3. High-dispersion coronagraphy (HDC) optimally combines high contrast imaging techniques such as adaptive optics/wavefront control plus coronagraphy to high resolution spectroscopy. The ultimate goal is to suppress the star light, thus reducing the photon noise so that a high-resolution spectrum of the planet can be extracted. This is achieved firstly by utilizing a high performance coronagraph and secondly by using a fiber in a downstream focal plane to selectively couple light from the planet in that region of the image plane to the spectrograph. ${ }^{4-6}$ The KPIC instrument will take this one step further by specifically employing single mode fibers. It was recently shown in Ref. 1 that due to the properties of such fibers (i.e. that they only allow light to propagate down the fiber which conforms to the near-Gaussian illumination profile and flat phase front conditions) the complex field of the star light can be modulated with an upstream deformable mirror in order to selectively prevent it from coupling into these fibers. This technique will offer further possibilities of reducing photon noise and will be tested on the KPIC instrument in the future.

HDC is a critical pathway towards fully characterizing exoplanet atmospheres across a broad range of masses from giant gaseous planets down to Earth-like planets in the future with ELTs and space telescopes. ${ }^{2}$ In addition to determining the molecular composition of exoplanet atmospheres, ${ }^{7,8} \mathrm{HDC}$ also enables Doppler mapping ${ }^{9}$ of atmosphere inhomogeneities (temperature, clouds, wind), as well as precise measurements of exoplanet rotational velocities. ${ }^{10}$ 


\section{FIBER INJECTION UNIT}

The FIU is shown in Fig. 3 and consists of a series of optical relays, a tip-tilt mirror, a beamsplitter, a bundle of single mode fibers, a corner cube and a tracking camera. Located downstream from the coronagraph, the FIU is fed by a collimated beam. The light is reflected by a tip-tilt mirror in the direction of a dichroic dividing the light into two beams. The first one is focused on the tracking camera and the second one on the fiber bundle. The image formed on the tracking camera allows for the star position to be determined, which will be used to guide on while spectroscopy of the known exoplanet is underway.

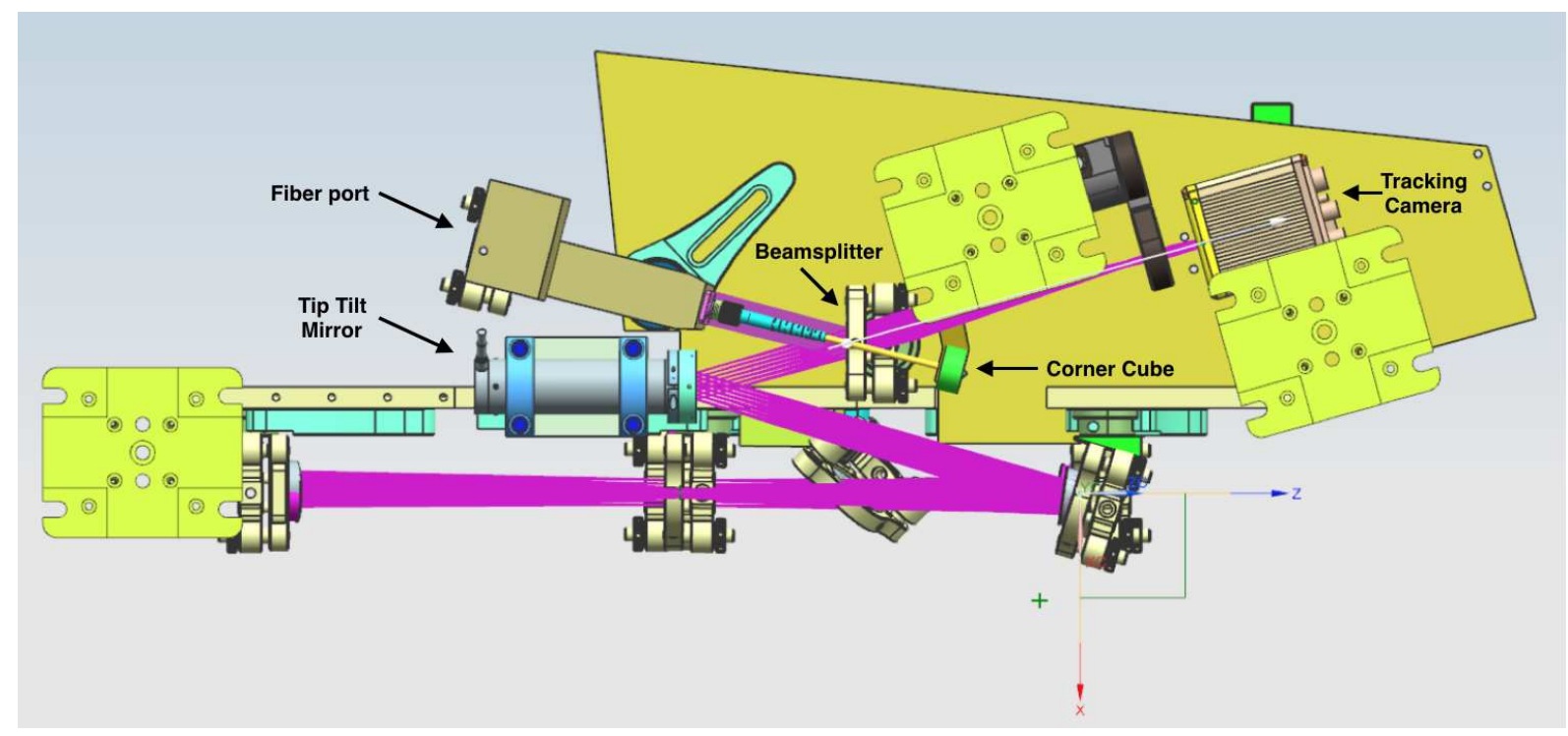

Figure 3. Bottom view of KPIC where the FIU is visible.

To determine the relative position of the fibers in the bundle to the star/planet, one or several fibers in the bundle are retro-fed by a laser source. This light, collimated by the optic in front of the bundle, goes through the dichroic before being reflected by the corner cube and then being steered by the dichroic onto the tracking camera. Assuming the relative position of each fiber of the bundle is calibrated a priori, one can determine the position of the star/planet with respect to the fibers. To inject the planet light into the fiber, we use the tip-tilt mirror to move the planet to the position of the science fibers. The bundle is composed of eight single mode fibers. Five of them are connected to NIRSPEC and can be used as science fibers while the other three ones are connected to the laser diode and can be retro-fed in order to be used as reference fibers. In order to properly reduce the data, two fibers are allocated to monitoring the starlight and sky background.

\subsection{Requirements}

The KPIC FIU shall transport the $\mathrm{K}(\sim 2.0-2.4 \mu \mathrm{m})$ and L-band $(\sim 2.9-4.2 \mu \mathrm{m})$ light from the Keck II adaptive optics bench to NIRSPEC by means of single-mode fibers. Three fibers are needed to properly sample the exoplanet signal (fiber 1), the starlight for telluric absorption line removal (fiber 2), and the sky background for telluric emission lines (fiber 3). The injection efficiency of the FIU for a diffraction-limited beam shall be larger than 50\%. Acquisition and tracking shall be performed with a dedicated sensor operating at shorter wavelengths, $\mathrm{J}(\sim 1.15-1.35 \mu \mathrm{m})$ and $\mathrm{H}$ band $(\sim 1.49-1.8 \mu \mathrm{m})$ by means of a dichroic beamsplitter. The tracking rates shall be limited by photon and background noise at those bands. Detector spatial sampling will be set for Nyquist sampling at $\mathrm{J}$ band (10 mas pixels). The requirement on tracking accuracy is based on achieving a high injection efficiency, which requires reducing tip/tilt to be better than $0.1 \lambda / D$ at the science wavelengths (K and L bands) over timescales of a few hours and for both position and vertical angle modes. The nominal unvignetted fieldof-view through the sensor will be about 7 arcseconds in diameter. The patrol area for the off-axis planet fiber around the central star will be of about 2 arcseconds. The sky background fiber will be maintained 3 arcseconds away from the central star. 


\subsection{Optical design and optics}

The FIU optical design is based on off-axis parabolas (OAP) arranged in a double symmetric F/15 relay as shown in Fig. 2. The OAPs were provided by Nu-Tek Precision Optics. Requirements for the surface figure per OAP is $15 \mathrm{~nm}$ rms. The first relay is designed to accommodate a Boston MicroMachines 34x34 actuators, $3.5 \mu \mathrm{m}$ stroke deformable mirror, and a beamsplitter sending the light to an infrared pyramid wavefront sensor. The second relay is designed to accommodate a reflective pupil apodizer and a possible focal plane coronagraphic mask. The third relay takes the Keck F/15 beam through a beamsplitter creating two foci: one on the tracking camera and another one on the fiber bundle. The end-to-end wavefront quality of the FIU is better than $60 \mathrm{~nm}$ rms.

\subsection{Tip-tilt mirror}

The tip-tilt mirror is used to position the science target on the fiber array, and track. We chose a strain-gauge closed-loop control fast steering mirror from the S-330 series from Physik Instrumente. This mirror satisfies our field of regard (off-axis planet fiber patrol area) and tracking accuracy requirements.

\subsection{Tracking camera}

The tracking camera will be fast and low-noise and operate at both $\mathrm{J}$ and $\mathrm{H}$ bands. We chose to use the C-RED2 from First Light Imaging (FLI). The C-RED 2 is a 640x512 InGaAs camera with the following characteristics: InGaAs 640 x 512 PIN Photodiode sensor, $0.9 \mu \mathrm{m}$ to $1.7 \mu \mathrm{m}, 70 \%$ Quantum Efficiency $15 \mu \mathrm{m}$ pixel pitch, windowing and ROI modes available, TE3 cooled sensor operation for low dark current. The camera can provide very low read noise $\left(<30 e^{-}\right)$and dark current $\left(100 e^{-} /\right.$pixel $\left./ s\right)$ at high frame rates (up to $400 \mathrm{~Hz}$ full frame). The camera is also compact, which integrates well in our tight operating space constraints.

\subsection{Fiber port}

The KPIC FIU prototype (first plate) will contain only one fiber port. This custom part shown in Fig. 4, contains an off-axis parabola (OAP) in a three-axis mount and a connector for the fiber bundle. The focal length of the OAP is optimized to maximize the light injection into the fiber of the bundle described in the next paragraph.

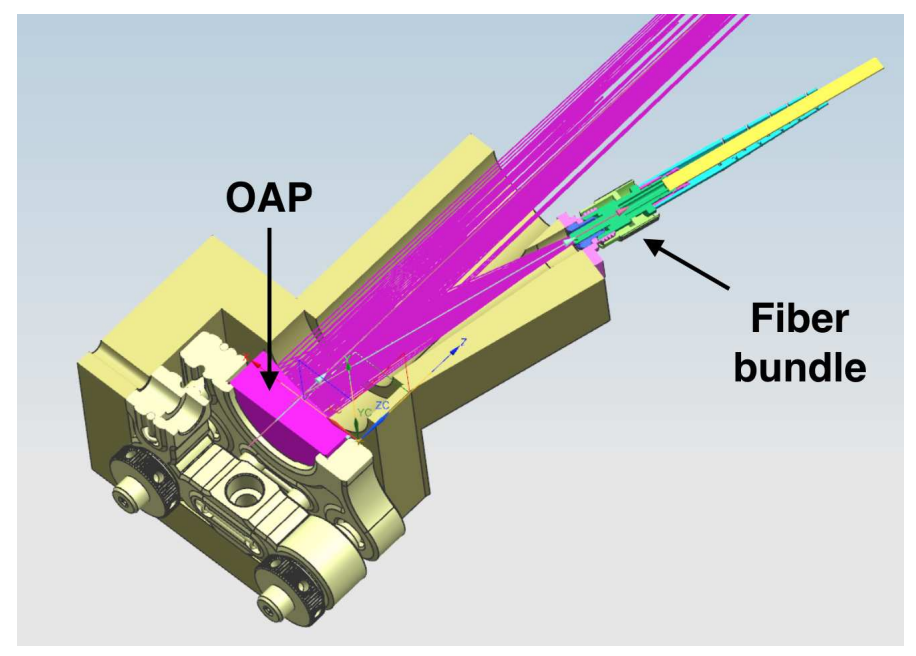

Figure 4. Cross section of the first version fiber port design for the FIU of KPIC.

The second iteration of the FIU will have three output ports to accommodate several fiber bundle designs. The additional ports will enable other astronomical applications including a mini micro-lens based integral-field unit, as well as an infrared precision radial velocity feed calibrated with a laser Frequency Comb. 


\subsection{Bundle design}

The first fiber bundle used to connect KPIC to NIRSPEC is made of eight ZBLAN $6.5 \mu \mathrm{m}$ core, $125 \mu \mathrm{m}$ cladding off-the-shelf fluoride fibers manufactured by Le Verre Fluoré that offer a single mode cut-off at $1.95 \mu \mathrm{m}$. Fig. 5 presents a schematic diagram of the bundle. In the input side, which connects to the FIU (left), the eight fibers are butted against each other cladding to cladding. In this case, the cores of the fibers are separated by $125 \mu \mathrm{m}$ which corresponds to an angular separation of 0.89 arcsecond on sky. The three fibers marked in orange will be used for alignment of the bundle with respect to the star/planet system while the five blue fibers will be used permanently for science. The output end of the alignment fibers are connected to laser diodes while the output end of the science fibers are connected to NIRSPEC. In this connector (right), the five fibers are also aligned cladding to cladding.

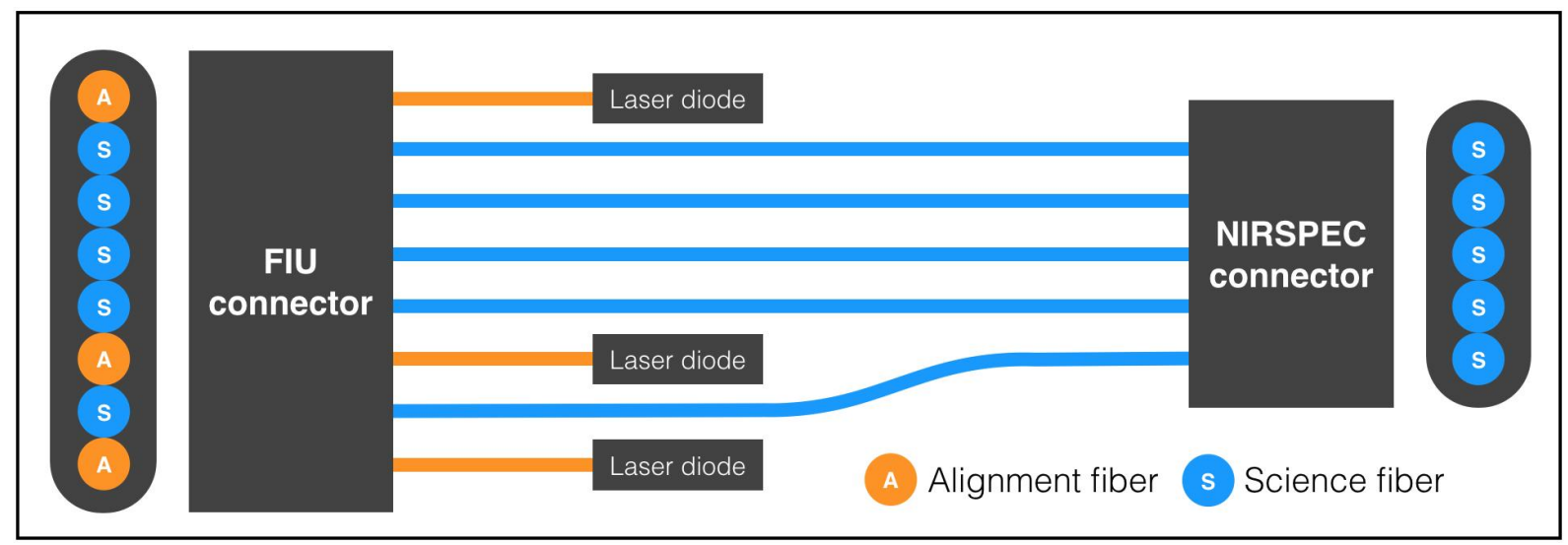

Figure 5. Diagram of the first fiber bundle designed for KPIC.

Before the observations, the alignment fibers will be retro-fed by the laser diodes in order to determine the position of the fibers in the FIU side. Then during observations, the fibers will be oriented with the star-planet system plane using the K-mirror of the Keck II AO. One of the science fibers will be fed by the planetary light using the tip-tilt mirror while two of the four other science fibers will be allocated to starlight and sky background measurements in order to properly reduce the data.

\subsection{Fiber Extraction Unit}

The Fiber Extraction Unit (FEU) will be used to relay the light from the output of the fiber bundle onto the slit of the NIRSPEC spectrograph. The basic requirements are that the mode field diameter of the science fibers will be matched to the width of the 0.288 " slit in NIRSPEC. This offers three pixels of sampling at the detector. With this arrangement a maximum of five fibers can fit along the length of the slit. This is where this requirement comes from for the bundle. The slit will truncate the fiber modes and the rejected light will be reflected to a H2RG detector for monitoring the flux coupled into the fibers as well as tracking any misalignment's between the fiber bundle and the slit. This will be critical for precision radial velocity work.

To achieve this, the beam emanating from the output of the fibers must be collimated with a lens or OAP to a radius of $8.2 \mathrm{~mm}$ and carefully controlled. The design of such an optical system is currently underway and we plan to include the ability to steer both the field and pupil with very simple mechanics.

\section{CONCLUSION}

We presented the latest iteration of the KPIC fiber injection unit design and its status. We decided to build the FIU in two stages, based on the same double relay concept. The first stage FIU will be integrated without the deformable mirror and coronagraph option over the next few months (Fall of 2017), and shipped to Keck Observatory for a first demonstration on sky in 2018. In parallel, a second FIU plate will be built, mostly identical to the first one but equipped with a deformable mirror and coronagraph module. We expect the second plate to be ready by the end of 2018 and be shipped and commissioned at Keck Observatory in 2019. 


\section{ACKNOWLEDGMENTS}

The authors would like to acknowledge the financial support of the Heising-Simons foundation. This work was partially carried out at the Jet Propulsion Laboratory, California Institute of Technology, under contract with the

National Aeronautics and Space Administration. Finally, we would like to thank Rowan Swain for proofreading the manuscript.

\section{REFERENCES}

1. D. Mawet, G. Ruane, W. Xuan, D. Echeverri, N. Klimovich, M. Randolph, J. Fucik, J. K. Wallace, J. Wang, G. Vasisht, R. Dekany, B. Mennesson, E. Choquet, J.-R. Delorme, and E. Serabyn, "Observing Exoplanets with High-dispersion Coronagraphy. II. Demonstration of an Active Single-mode Fiber Injection Unit," ApJ 838(2), p. 92, 2017.

2. J. Wang, D. Mawet, G. Ruane, R. Hu, and B. Benneke, "Observing Exoplanets with High Dispersion Coronagraphy. I. The Scientific Potential of Current and Next-generation Large Ground and Space Telescopes," AJ 153, p. 183, Apr. 2017.

3. I. Snellen, R. de Kok, J. L. Birkby, B. Brandl, M. Brogi, C. Keller, M. Kenworthy, H. Schwarz, and R. Stuik, "Combining high-dispersion spectroscopy with high contrast imaging: Probing rocky planets around our nearest neighbors," A\&A 576, p. A59, Apr. 2015.

4. N. Jovanovic, C. Schwab, N. Cvetojevic, O. Guyon, and F. Martinache, "Enhancing Stellar Spectroscopy with Extreme Adaptive Optics and Photonics," 128, p. 121001, Dec. 2016.

5. N. Jovanovic, N. Cvetojevic, B. Norris, C. Betters, C. Schwab, J. Lozi, O. Guyon, S. Gross, F. Martinache, P. Tuthill, D. Doughty, Y. Minowa, N. Takato, and J. Lawrence, "Demonstration of an efficient, photonicbased astronomical spectrograph on an 8-m telescope," ArXiv e-prints, July 2017.

6. N. Jovanovic, C. Schwab, O. Guyon, J. Lozi, N. Cvetojevic, F. Martinache, S. Leon-Saval, B. Norris, S. Gross, D. Doughty, T. Currie, and N. Takato, "Efficient injection from large telescopes into single-mode fibres: Enabling the era of ultra-precision astronomy," ArXiv e-prints, June 2017.

7. Q. M. Konopacky, T. S. Barman, B. A. Macintosh, and C. Marois, "Detection of Carbon Monoxide and Water Absorption Lines in an Exoplanet Atmosphere," Science 339, pp. 1398-1401, Mar. 2013.

8. T. S. Barman, Q. M. Konopacky, B. Macintosh, and C. Marois, "Simultaneous Detection of Water, Methane, and Carbon Monoxide in the Atmosphere of Exoplanet HR8799b," 804, p. 61, May 2015.

9. I. J. M. Crossfield, B. Biller, J. E. Schlieder, N. R. Deacon, M. Bonnefoy, D. Homeier, F. Allard, E. Buenzli, T. Henning, W. Brandner, B. Goldman, and T. Kopytova, "A global cloud map of the nearest known brown dwarf," Nature 505, pp. 654-656, Jan. 2014.

10. I. A. G. Snellen, B. R. Brandl, R. J. de Kok, M. Brogi, J. Birkby, and H. Schwarz, "Fast spin of the young extrasolar planet $\beta$ Pictoris b," Nature 509, pp. 63-65, May 2014. 